\section{Complementing mAbs}

\section{By Kai-Jye Lou, Senior Writer}

Genmab A/S and Utrecht University researchers have shown that hexameric IgG complexes can activate the complement cascade, providing the molecular rationale behind the biotech's HexaBody platform. ${ }^{1}$ Genmab is now selecting key mutations and antibody candidates to generate IgG antibodies that potently induce complementdependent cytotoxicity in cancer and infectious disease.

In complement-dependent cytotoxicity, antigen-bound antibodies activate the complement system to induce the formation of membrane attack complexes on the surface of the target cell. These complexes disrupt the integrity of the cell membrane, resulting in lysis.

Despite being a well-known phenomenon, the mechanistic details of how antibodies activate the complement system were poorly characterized.

Genmab presented preliminary proof-of-concept efficacy data for its HexaBody technology at the American Society of Hematology meeting last December. In preclinical leukemia and lymphoma models, IgG antibodies with mutations in the Fc region conferring an enhanced ability to form hexamer structures induced more potent complementdependent cytotoxicity than unmodified counterparts.

The IgG hexamers are arranged in a ring structure, with the Fc regions of the antibodies pointed toward the center.

At the time, the company did not disclose the mechanistic underpinnings of how enhancing IgG hexamer formation with its HexaBody platform led to improved complement-dependent cytotoxicity.

Now, a group co-led by Utrecht University and Genmab researchers has published in Science the details of how IgGs activate the complement system.

The researchers used a blocking peptide and a series of point mutation studies to show that the ability of IgGs to form hexamers is mediated by noncovalent interactions involving the antibody's Fc region. Disrupting the antibody's ability to form hexamers had multiple effects. These included impaired recruitment and activation of complement component $1 \mathrm{q}$ subcomponent $(\mathrm{C} 1 \mathrm{q})$ and decreased ability to induce complement-dependent cytotoxicity.

The researchers then showed that IgGs could be engineered to have enhanced hexamer-forming capabilities. The group engineered prototypical human mAbs from all four IgG subclasses with an E345R point mutation, which is located in the Fc region of IgG.

The mutation improved the antibody's ability to form hexamers upon binding a target antigen. These IgGs formed hexamers that recruited and potently activated C1q (see Figure 1, "Bird's-eye view of IgG hexamers").
In a human lymphoma cell line, the E345R-mutant IgGs were better able to activate the complement system and induce complement-dependent cytotoxicity than unmodified isotype controls.

Researchers from The Scripps Research Institute also contributed to the study.

"We showed that Fc-Fc contacts are indeed important for the assembly of these antibodies into hexamer structures after they've bound to their target antigen and that it is this hexamer that acts as the docking structure for C1q," said Genmab SVP and scientific director Paul Parren, the principal investigator on the study. "Now that we know how to enhance this hexamer-forming activity, we could potentially engineer antibodies that induce improved killing of target cells, and hopefully that will translate into improved therapeutic efficacy."

"The demonstration that IgG hexamerization after antigen binding is effective for all four human IgG isotypes is particularly relevant and

Figure 1. Bird's-eye view of IgG hexamers. The complement component $1 \mathrm{q}$ subcomponent $(\mathrm{C} 1 \mathrm{q})$ complex has six antibody-binding headpieces (blue circles) and activates the complement system. Antigen-bound antibodies recruit and activate $\mathrm{C} 1 \mathrm{q}$, but individual $\lg \mathrm{G}$ antibody molecules bind the complex with weak affinity.

As reported by Diebolder et al., monoclonal IgGs can be engineered with point mutations that enhance their ability to assemble into hexamers after binding to a target antigen on the cell surface.

This IgG hexamer structure is better able to recruit and activate the $\mathrm{C} 1 \mathrm{q}$ complex, leading to increased complement activation and more potent complement-dependent cytotoxicity against the target cell compared with individual lgG molecules.

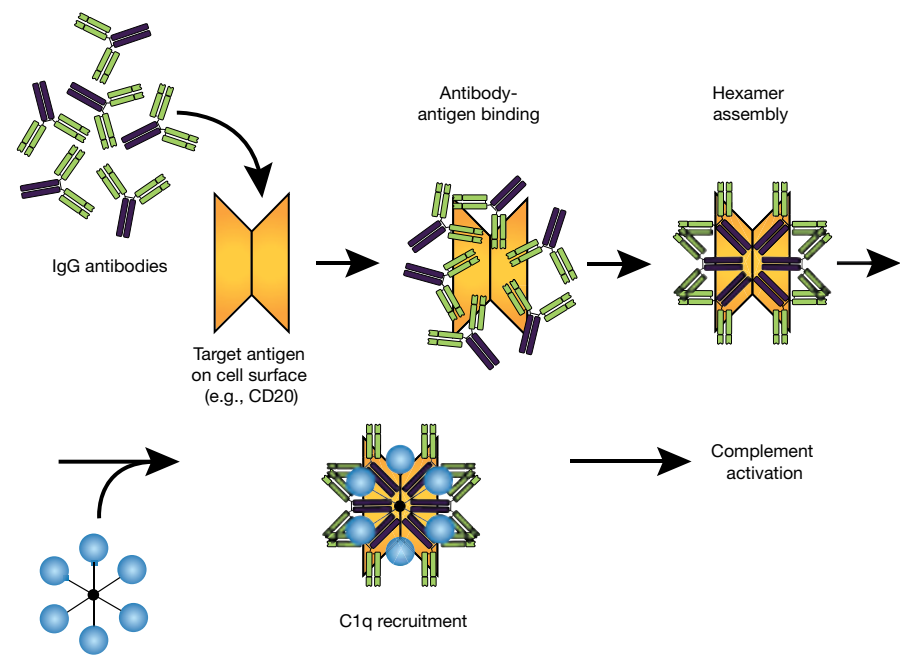

C1q complex 
convincing," said Alain Beck, senior director of antibody and antibody-drug conjugate physico-chemistry at the Center of Immunology Pierre Fabre.

He said that the approach thus seems to be generalizable to designing immunotherapeutics with enhanced properties.

"At the atomic level, the findings provide insights on how to dissect and potentially modulate the amount of complement activation," said Barry Springer, VP of technology, strategy and operations for the Biotechnology Center of Excellence at the Janssen Research \& Development LLC unit of Johnson \& Johnson.

In 2012, Janssen Biotech Inc. partnered with Genmab to use the biotech's DuoBody technology to create panels of bispecific antibodies against undisclosed disease target combinations selected by Janssen.

Also that year, Genmab granted Janssen exclusive worldwide rights to develop and commercialize daratumumab (HuMax-CD38), a human $\mathrm{mAb}$ against $\mathrm{CD} 38$, for $\$ 55$ million up front, up to $\$ 1$ billion in milestones and tiered, double-digit royalties. The pharma also took a $10.7 \%$ percent equity stake in Genmab.

The pharma plans to start Phase III testing of daratumumab to treat relapsed or refractory multiple myeloma (MM) this month.

The pharma does not have a partnership involving HexaBody technology.

\section{Bolstering an insufficient response}

A key goal of Genmab's HexaBody technology is to provide a general approach for enhancing the potency of new or existing mAbs without significantly disrupting their native structure and specificity.

Parren and Springer both noted that engineering mAbs with an improved ability to induce complement-dependent cytotoxicity could be useful in situations in which the cell-killing response caused by an existing antibody is insufficient.

Parren said that HexaBody technology could be used to augment the effect of existing therapeutic mAbs that kill target cells via other mechanisms, such as the blockade of a key receptor or antibodydependent cell-mediated cytotoxicity.

"Some pathogen and tumor cell antigens have low presentation or density on the cell surface, which could limit the ability of native antibodies" to kill the cell, added Springer. "The current study suggests they could now potentially bypass such issues by specifically tailoring antibodies that have a propensity to aggregate and promote complex formation."

According to Parren, the mechanism described in the paper suggests that
mAbs engineered with the HexaBody platform would induce complementdependent cytotoxicity in a highly specific and localized manner.

"The hexamer formation process only happens once the antibody binds to the target antigen. The IgGs otherwise remain in a monomeric state while in circulation that cannot interact with C1q," he said.

Parren declined to disclose the specific complement-enhancing mutations and mAbs that Genmab plans to use for its HexaBody platform but did note that the E345R mutation described in the study is just a prototype complement-enhancing mutation and not one of the mutations the company expects to use.

"The Science paper helps to set the stage for others to understand what the potential of our HexaBody technology could be. We are definitely thinking about how to use this technology to generate new candidates for our pipeline," he added.

Springer said that it will be important to determine the extent to which enhancing complement activity could promote tumor cell killing and how incorporating improved complement activation affects therapeutic mAbs that already kill their target cells via other antibody-mediated mechanisms such as antibody-dependent cell-mediated cytotoxicity.

He added that it also will be important to show that it is possible to do scaled-up manufacturing of the complement-activating mAbs.

Genmab has filed multiple patents covering various aspects of its HexaBody technology. The technology is available for licensing and partnering.

Lou, K.-J. SciBX 7(13); doi:10.1038/scibx.2014.363

Published online April 3, 2014

\section{REFERENCES}

1. Diebolder, C.A. et al. Science; published online March 13, 2014; doi:10.1126/science.1248943

Contact: Paul W.H.I. Parren, Genmab A/S, Utrecht, the Netherlands e-mail: p.parren@genmab.com

Contact: Piet Gros, Utrecht University, Utrecht, the Netherlands e-mail: p.gros@uu.nl

\section{COMPANIES AND INSTITUTIONS MENTIONED}

American Society of Hematology, Washington, D.C. Center of Immunology Pierre Fabre,

St. Julien-en-Genevois, France

Genmab A/S (CSE:GEN; OTCBB:GMXAY), Copenhagen, Denmark

Johnson \& Johnson (NYSE:JNJ), New Brunswick, N.J.

The Scripps Research Institute, La Jolla, Calif.

Utrecht University, Utrecht, the Netherlands 